\title{
Some Results on Cyclic Interval Edge Colorings of Graphs
}

\author{
Armen S. Asratian ${ }^{a *}$, Carl Johan Casselgren ${ }^{a \dagger}$, Petros A. Petrosyan $^{\mathrm{bc} \ddagger}$ \\ ${ }^{a}$ Department of Mathematics, Linköping University, \\ SE-581 83 Linköping, Sweden \\ ${ }^{\mathrm{b}}$ Department of Informatics and Applied Mathematics, \\ Yerevan State University, 0025, Armenia \\ ${ }^{\mathrm{c}}$ Institute for Informatics and Automation Problems, \\ National Academy of Sciences, 0014, Armenia
}

A proper edge coloring of a graph $G$ with colors $1,2, \ldots, t$ is called a cyclic interval $t$-coloring if for each vertex $v$ of $G$ the edges incident to $v$ are colored by consecutive colors, under the condition that color 1 is considered as consecutive to color $t$. We prove that a bipartite graph $G$ of even maximum degree $\Delta(G) \geq 4$ admits a cyclic interval $\Delta(G)$-coloring if for every vertex $v$ the degree $d_{G}(v)$ satisfies either $d_{G}(v) \geq \Delta(G)-2$ or $d_{G}(v) \leq 2$. We also prove that every Eulerian bipartite graph $G$ with maximum degree at most 8 has a cyclic interval coloring. Some results are obtained for $(a, b)$-biregular graphs, that is, bipartite graphs with the vertices in one part all having degree $a$ and the vertices in the other part all having degree $b$; it has been conjectured that all these have cyclic interval colorings. We show that all $(4,7)$-biregular graphs as well as all $(2 r-2,2 r)$ biregular $(r \geq 2)$ graphs have cyclic interval colorings. Finally, we prove that all complete multipartite graphs admit cyclic interval colorings; this proves a conjecture of Petrosyan and Mkhitaryan.

Keywords: edge coloring, interval coloring, cyclic interval coloring, bipartite graph, biregular graph, complete multipartite graph.

\section{Introduction}

We use 29] for terminology and notation not defined here. All graphs considered are finite, undirected, allow multiple edges and contain no loops, unless otherwise stated. A simple graph is a graph with no loops or multiple edges. Let $V(G)$ and $E(G)$ denote the sets of vertices and edges of a graph $G$, respectively. A proper $t$-edge coloring of a graph $G$ is a mapping $\alpha: E(G) \longrightarrow\{1, \ldots, t\}$ such that $\alpha(e) \neq \alpha\left(e^{\prime}\right)$ for every pair of adjacent edges $e$ and $e^{\prime}$ in $G$. If $e \in E(G)$ and $\alpha(e)=k$ then we say that the edge $e$ is colored $k$. We

*email: armen.asratian@liu.se

†email: carl.johan.casselgren@liu.se

†email: petros_petrosyan@ysu.am, pet_petros@ipia.sci.am 
denote by $\Delta(G)$ the maximum degree of vertices of a graph $G$, and by $d_{G}(v)$ the degree of a vertex $v$ in $G$. The chromatic index $\chi^{\prime}(G)$ of a graph $G$ is the minimum number $t$ for which there exists a proper $t$-edge coloring of $G$. By Kőnig's edge coloring theorem, $\chi^{\prime}(G)=\Delta(G)$ for any bipartite graph $G$ and by Vizing's theorem $\chi^{\prime}(G) \leq \Delta(G)+1$ for any simple graph $G$ (see for example [29]).

A proper $t$-edge coloring of a graph $G$ is called an interval $t$-coloring if the colors of edges incident to every vertex $v$ of $G$ form an interval of integers. This notion was introduced by Asratian and Kamalian [5] (available in English as [6]), motivated by the problem of constructing timetables without gaps for teachers and classes. Later the theory of interval colorings was developed in e.g. [3,4,7,8,9,10,11,12,13,14,15,21,22,23, 25, 27, 30]. Generally, it is an NP-complete problem to determine whether a bipartite graph has an interval coloring [27]. However some classes of graphs have been proved to admit interval colorings. It is known, for example, that trees, regular and complete bipartite graphs [5]11],13], doubly convex bipartite graphs [14], grids [9] and simple outerplanar bipartite graphs [10] have interval colorings. Additionally, all $(2, b)$-biregular graphs [11,12,15] and $(3,6)$-biregular graphs [8] admit interval colorings, where an $(a, b)$-biregular graph is a bipartite graph where the vertices in one part all have degree $a$ and the vertices in the other part all have degree $b$.

Another type of proper $t$-edge colorings, a cyclic interval $t$-coloring, was introduced by de Werra and Solot [28]. A proper $t$-edge coloring $\alpha: E(G) \longrightarrow\{1, \ldots, t\}$ of a graph $G$ is called a cyclic interval t-coloring if the colors of edges incident to every vertex $v$ of $G$ either form an interval of integers or the set $\{1, \ldots, t\} \backslash\{\alpha(e): e$ is incident to $v\}$ is an interval of integers. This notion was motivated by scheduling problems arising in flexible manufacturing systems, in particular the so-called cylindrical open shop scheduling problem. Clearly, any interval $t$-coloring of a graph $G$ is also a cyclic interval $t$-coloring. Therefore all above mentioned classes of graphs which admit interval edge colorings, also admit cyclic interval colorings. Note that the condition $\chi^{\prime}(G)=\Delta(G)$ is necessary for a graph $G$ to admit an interval edge coloring [5]6]. In contrast with this, every regular graph $G$ with $\chi^{\prime}(G)=\Delta(G)+1$ (for example, $G=K_{2 n+1}$ ) has a cyclic interval $(\Delta(G)+1)$ coloring. Moreover, for every integer $p \geq 1$ there is a graph $G_{p}$ with $\chi^{\prime}(G)=\Delta(G)+p$ which admits a cyclic interval coloring. An example of such a graph is the so-called "Shannon's triangle" which is obtained by replacing every edge in a triangle $K_{3}$ with $V\left(K_{3}\right)=\left\{v_{1}, v_{2}, v_{3}\right\}$ by $p$ parallel edges. Clearly, the maximum degree of this graph is $2 p$, the chromatic index is $3 p$, and a cyclic interval coloring of it can be obtained by coloring the edges between $v_{k}$ and $v_{k+1}$ with colors $(k-1) p+1,(k-1) p+2, \ldots,(k-1) p+p$, for $k=1,2,3$ (where we consider $v_{4}=v_{1}$ ).

Note further that if a graph has a cyclic interval $t$-coloring, then it does not necessarily have a cyclic interval $(t+1)$-coloring; the complete graph $K_{3}$ has a cyclic interval 3coloring, but does not admit such a coloring with 4 colors (see also Example 4.5 in Section 4). Furthermore, the disjoint union of graphs with cyclic interval colorings may not admit a cyclic interval coloring; for instance, the disjoint union of $K_{3}$ and $K_{n}$ does not have a cyclic interval coloring for any $n \geq 5$.

Kubale and Nadolski [18] showed that the problem of determining whether a given bipartite graph admits a cyclic interval coloring is $N P$-complete. Some sufficient conditions for a graph to have a cyclic interval coloring were obtained in [8,19,24,28]. Nadolski [19] 
proved that any connected graph $G$ with $\Delta(G) \leq 3$ has a cyclic interval coloring. de Werra and Solot [28] proved that any outerplanar bipartite graph $G$ has a cyclic interval $\Delta(G)$-coloring. Petrosyan and Mkhitaryan [24] showed that all complete tripartite graphs are cyclically interval colorable and conjectured that the same holds for all complete multipartite graphs. They also proved that if a triangle-free simple graph $G$ with at least two vertices has a cyclic interval $t$-coloring, then $t \leq|V(G)|+\Delta(G)-2$. Casselgren and Toft [8] proved that all (4,8)-biregular graphs admit cyclic interval colorings, and conjectured that the same holds for all $(a, b)$-biregular graphs. Some other results on this subject were obtained in [21617]. For example, Altinakar et al. [2] showed that for any graph $G$ with $\Delta(G) \geq 12$ there is a graph $H_{G}$ with $\Delta\left(H_{G}\right)=\Delta(G)$ such that $G$ has an interval $t$-coloring if and only if $H_{G}$ has a cyclic interval $t$-coloring. In [1617, Kamalian determined all possible values of $t$ for which simple cycles and trees have a cyclic interval $t$-coloring.

In the present paper we find new classes of graphs admitting cyclic interval colorings. We prove that a bipartite graph $G$ with even maximum degree $\Delta(G) \geq 4$ admits a cyclic interval $\Delta(G)$-coloring if for every vertex $v$ the degree $d_{G}(v)$ satisfies either $d_{G}(v) \geq$ $\Delta(G)-2$ or $d_{G}(v) \leq 2$. We also prove that every Eulerian bipartite graph $G$ with maximum degree $\Delta(G) \leq 8$ has a cyclic interval $\Delta(G)$-coloring. Furthermore, some results are obtained for $(a, b)$-biregular and outerplanar graphs. Finally, we prove that all complete multipartite graphs admit cyclic interval colorings and consider some problems on bipartite graphs without cyclic interval colorings.

\section{Cyclic interval colorings of bipartite graphs}

Before we formulate and prove our results, we introduce some terminology and notation. A graph $G$ is cyclically interval colorable if it has a cyclic interval $t$-coloring for some positive integer $t$. The set of all interval cyclically colorable graphs is denoted by $\mathfrak{N}_{c}$. For a graph $G \in \mathfrak{N}_{c}$, the least value of $t$ for which it has a cyclic interval $t$-coloring is denoted by $w_{c}(G)$. If $\alpha$ is a proper edge coloring of $G$ and $v \in V(G)$, then $S_{G}(v, \alpha)($ or $S(v, \alpha)$ ) denotes the set of colors appearing on edges incident to $v$.

For two positive integers $a$ and $b$, we denote by $\operatorname{gcd}(a, b)$ the greatest common divisor of $a$ and $b$; if $a \leq b$, then $[a, b]$ denotes the interval $\{a, \ldots, b\}$ of integers.

A full subdivision of a graph is a graph obtained by replacing each edge with a path of length 2. A graph $G$ is Eulerian if the degree of every vertex of $G$ is even. Note that if $G$ is connected and Eulerian, then it has a closed trail containing every edge of it. A 2 -factor of a graph $G$, where loops are allowed, is a 2-regular spanning subgraph of $G$. We need the following classical result from factor theory [1].

Petersen's theorem. Let $G$ be a $2 r$-regular graph (where loops are allowed). Then $G$ can be represented as a union of edge-disjoint 2-factors.

The main result of this section is the following:

Theorem 2.1 If $G$ is a bipartite graph with $\Delta(G)=2 r(r \geq 2)$ and for every $v \in V(G)$, $d_{G}(v) \in\{1,2,2 r-2,2 r-1,2 r\}$, then $G$ has a cyclic interval $2 r$-coloring. 
Proof. Define an auxiliary graph $G^{\star}$ as follows: first we take two isomorphic copies $G_{1}$ and $G_{2}$ of the graph $G$ and join by an edge every vertex with an odd vertex degree in $G_{1}$ with its copy in $G_{2}$, then for each vertex $u \in V\left(G_{1}\right) \cup V\left(G_{2}\right)$ of degree 2 , we add $r-1$ loops at $u$, and for each vertex $v \in V\left(G_{1}\right) \cup V\left(G_{2}\right)$ of degree $2 r-2$, we add a loop at $v$. Clearly, $G^{\star}$ is a $2 r$-regular graph. By Petersen's theorem above, $G^{\star}$ can be represented as a union of edge-disjoint 2-factors $F_{1}, \ldots, F_{r}$. By removing all loops from 2-factors $F_{1}, \ldots, F_{r}$ of $G^{\star}$, we obtain that the resulting graph $G^{\prime}$ is a union of edge-disjoint Eulerian subgraphs $F_{1}^{\prime}, \ldots, F_{r}^{\prime}$. Since $G^{\prime}$ is bipartite, for each $i(1 \leq i \leq r), F_{i}^{\prime}$ is a collection of even cycles in $G^{\prime}$, and we can color the edges of $F_{i}^{\prime}$ alternately with colors $2 i-1$ and $2 i$. Let $\alpha$ be the resulting coloring of $G^{\prime}$. Clearly, $\alpha$ is a proper edge coloring of $G^{\prime}$ with colors $1, \ldots, 2 r$, and for each vertex $v \in V\left(G^{\prime}\right)$ with $d_{G^{\prime}}(v)=2 r, S_{G^{\prime}}(v, \alpha)=[1,2 r]$. Since for each vertex $u \in V\left(G^{\prime}\right)$ with $d_{G^{\prime}}(u)=2$, there exists exactly one Eulerian subgraph $F_{i_{u}}^{\prime}$ such that $d_{F_{i_{u}}^{\prime}}(u)=2$, we obtain that $S_{G^{\prime}}(u, \alpha)=\left[2 i_{u}-1,2 i_{u}\right]$ for some $i_{u}$. Similarly, since for each vertex $v \in V\left(G^{\prime}\right)$ with $d_{G^{\prime}}(v)=2 r-2$, there exists exactly one Eulerian subgraph $F_{i_{v}}^{\prime}$ such that $d_{F_{i,}^{\prime}}(v)=0$, we obtain that $S_{G^{\prime}}(v, \alpha)=[1,2 r] \backslash\left[2 i_{v}-1,2 i_{v}\right]$ for some $i_{v}$. Now we can consider the restriction of this proper edge coloring to the edges of the graph $G$. Clearly, this coloring is a cyclic interval $2 r$-coloring of $G$.

From Theorem 2.1 we deduce a number of corollaries.

Corollary 2.2 Let $H$ be a graph with $\Delta(H)=2 r(r \geq 2)$ where for every $v \in V(H)$ either $d_{H}(v) \geq 2 r-2$ or $d_{H}(v) \leq 2$ holds. Then a full subdivision of $H$ admits a cyclic interval $2 r$-coloring.

A direct consequence of Corollary 2.2 is the following:

Corollary 2.3 A full subdivision $G$ of a graph $H$ admits a cyclic interval $\Delta(G)$-coloring if the maximum degree $\Delta(H)$ is even and differs from the minimum degree of $H$ by at most 2.

In [23], Petrosyan and Khachatrian showed that if a bipartite graph is interval colorable, then a full subdivision of this graph is also interval colorable and conjectured that the same holds for all interval colorable graphs. Recently, Pyatkin [26] confirmed this conjecture.

Corollary 2.4 If $G$ is a bipartite graph with $\Delta(G)=4$, then $G \in \mathfrak{N}_{c}$ and $w_{c}(G)=4$.

Proof. Let $G$ be a bipartite graph with maximum degree 4 . Clearly, $d_{G}(v) \in\{1,2,3,4\}$ for every vertex $v \in V(G)$, which, by Theorem 2.1, means that $G$ admits a cyclic interval 4 -coloring.

Our next result concerns bipartite graphs with an odd maximum degree.

Theorem 2.5 If $G$ is a bipartite graph with $\Delta(G)=2 r-1(r \geq 2)$ and for every $v \in V(G), d_{G}(v) \in\{1,2,2 r-2,2 r-1\}$, then $G \in \mathfrak{N}_{c}$ and $w_{c}(G) \leq 2 r$. 
Proof. Let us construct an auxiliary graph $G^{\star}$ as follows: we take two isomorphic copies of the graph $G$ and join by an edge one vertex of degree $2 r-1$ with its copy. It is easy to see that $G^{\star}$ is a bipartite graph with $\Delta\left(G^{\star}\right)=2 r(r \geq 2)$ and for every $v \in V\left(G^{\star}\right)$, $d_{G^{\star}}(v) \in\{1,2,2 r-2,2 r-1,2 r\}$. By Theorem 2.1, $G^{\star}$ has a cyclic interval $2 r$-coloring. Now we can consider the restriction of this cyclic interval coloring to the edges of the $G$. This coloring is a cyclic interval coloring of $G$ with no more than $2 r$ colors. Hence, $G \in \mathfrak{N}_{c}$ and $w_{c}(G) \leq 2 r$.

Note that Theorems 2.1 and 2.5 imply that every bipartite graph where all vertex degrees are in the set $\{1,2,4,5,6\}$ has a cyclic interval edge coloring.

Before we move on, we need the following result on bipartite graphs.

Lemma 2.6 If $G$ is a bipartite graph with $\Delta(G)=4$ and with no vertices of degree 3 , then $G$ has an interval 4-coloring $\alpha$ such that for each $v \in V(G)$ with $d_{G}(v)=2$, either $S_{G}(v, \alpha)=[1,2]$ or $S_{G}(v, \alpha)=[3,4]$.

Proof. If $G$ has pendant vertices, then we can construct an auxiliary graph $G^{\prime}$ as follows: we take two isomorphic copies of $G$ and join by an edge every pendant vertex with its copy. It is easy to see that $G^{\prime}$ is a bipartite graph with $\Delta\left(G^{\prime}\right)=4$ and with no vertices of degree 1 or 3 . So, without loss of generality, we may assume that the degree of every vertex of $G$ is either 4 or 2 .

Next, we construct an auxiliary graph $G^{\star}$ with loops as follows: for each vertex $v \in V(G)$ with $d_{G}(v)=2$, we add a loop at $v$. Clearly, $G^{\star}$ is a 4-regular graph with loops. By Petersen's theorem above, $G^{\star}$ can be decomposed into two edge-disjoint 2-factors $F_{1}$ and $F_{2}$. By removing all loops from 2-factors $F_{1}$ and $F_{2}$ of $G^{\star}$, we obtain that $G$ can be decomposed into two edge-disjoint Eulerian subgraphs $F_{1}^{\prime}$ and $F_{2}^{\prime}$. Since $G$ is bipartite, for each $i(1 \leq i \leq 2), F_{i}^{\prime}$ is a collection of even cycles in $G$, and we can color the edges of $F_{i}^{\prime}$ alternately with colors $2 i-1$ and $2 i$. Let $\alpha$ be the resulting coloring of $G$. Clearly, $\alpha$ is a proper edge coloring of $G$ with colors $1,2,3,4$, and for each vertex $u \in V(G)$ with $d_{G}(u)=4, S_{G}(u, \alpha)=[1,4]$. Since for each $v \in V(G)$ with $d_{G}(v)=2$, either $d_{F_{1}^{\prime}}(v)=0$ or $d_{F_{2}^{\prime}}(v)=0$, we obtain that either $S_{G}(v, \alpha)=[3,4]$ or $S_{G}(v, \alpha)=[1,2]$.

It follows from Theorem 2.1 that every Eulerian bipartite graph of maximum degree at most 6 has a cyclic interval coloring. Next, we prove that a stronger proposition is true.

Theorem 2.7 Every Eulerian bipartite graph $G$ with maximum degree at most 8 has a cyclic interval $\Delta(G)$-coloring.

Proof. Let $G$ be an Eulerian bipartite graph with maximum degree at most 8 . If $G$ has maximum degree at most 6 , then the result follows from Theorem 2.1, so we may assume that $G$ has maximum degree 8. From $G$ we form a new graph $H$ by splitting each vertex $v$ of degree 6 into two new vertices $v^{\prime}$ and $v^{\prime \prime}$, where $v^{\prime}$ has degree 2 and $v^{\prime \prime}$ has degree 4. The partitioning of edges in this splitting is arbitrary, other then ensuring that each vertex receives the correct degree. Observe that the resulting graph $H$ is bipartite and the degree of each vertex in $H$ is either 2,4 or 8 . 
Note that some components of $H$ might only contain vertices of degree 2. Let $H^{\prime}$ be the subgraph of $H$ containing every component of $H$ where all vertices have degree 2 , and set $\hat{H}=H-V\left(H^{\prime}\right)$.

From $\hat{H}$ we form a new graph $K$ by replacing every maximal path, where all the internal vertices have degree 2 , by an edge joining the endpoints of the path; we call such a path in $\hat{H}$ reducible. In the resulting graph $K$ every vertex has degree 4 or 8 . Moreover, $K$ may contain loops (and multiple edges).

Since every vertex degree in $K$ is divisible by $4, K$ has an even number of edges, and so $K$ has an Eulerian trail $T$ with an even number of edges. (If $K$ contains loops, then we choose $T$ in such a way that all loops at a particular vertex $v$ are traversed the first time that we visit $v$.)

We color the edges of $T$ alternately with colors "Blue" and "Red" in such a way that every vertex is incident with equally many Red and Blue edges (where possible loops are counted twice).

Since every reducible path in $\hat{H}$ corresponds to a single edge in $K$, the edge coloring of $K$ defines an edge coloring of $\hat{H}$ in the following way:

- for every edge in $\hat{H}$ that is in $K$, we retain the color of this edge;

- for each reducible path $P$ in $\hat{H}$, color every edge in $P$ with the color of the corresponding edge of $K$.

Next, we extend this edge coloring to $H^{\prime}$ by coloring every edge in this graph by the color Red. Denote the obtained edge coloring of $H$ by $\varphi$.

It is straightforward to see that the coloring $\varphi$ of $H$ satisfies the following:

- every vertex of degree 2 in $H$ is incident with two edges of the same color;

- every vertex of degree 4 in $H$ is incident with two Red and two Blue edges;

- every vertex of degree 8 in $H$ is incident with four Red edges and four Blue edges.

Furthermore, since there is a one-to-one correspondence between edges of $G$ and $H$, the coloring $\varphi$ induces an edge coloring $\varphi^{\prime}$ of $G$ such that

- every vertex of degree 2 in $G$ is incident with two edges of the same color;

- every vertex of degree 4 in $G$ is incident with two Red and two Blue edges;

- every vertex of degree 6 in $H$ is incident with four Red edges and two Blue edges, or two Red edges and four Blue edges;

- every vertex of degree 8 in $H$ is incident with four Red edges and four Blue edges.

The Blue edges in $G$ induces a subgraph $G_{1}$ of $G$, and the Red edges in $G$ induces a subgraph $G_{2}$ of $G$; so $G$ is the edge-disjoint union of the graphs $G_{1}$ and $G_{2}$. Moreover, for each $G_{i}(i=1,2)$ :

- every vertex of degree 2 in $G$ has either degree 0 or 2 in $G_{i}$; 
- every vertex of degree 4 in $G$ has degree 2 in $G_{i}$;

- every vertex of degree 6 in $G$ has degree 4 or 2 in $G_{i}$

- every vertex of degree 8 in $G$ has degree 4 in $G_{i}$.

Hence each of the subgraphs $G_{1}$ and $G_{2}$ is a bipartite graph where every vertex has degree 4 or 2. By Lemma 2.6, each $G_{i}$ has an interval 4-coloring $f_{i}$ such that for every vertex $v$ of degree 2 in $G_{i}, S_{G_{i}}\left(v, f_{i}\right)=[1,2]$ or $S_{G_{i}}\left(v, f_{i}\right)=[3,4]$. From $f_{1}$ we define a new edge coloring $g_{1}$ of $G_{1}$ by replacing colors 3 and 4 by colors 5 and 6 , respectively; from $f_{2}$ we define a new edge coloring $g_{2}$ of $G_{2}$ by replacing colors 1 and 2 by colors 7 and 8 , respectively. It is straightforward to verify that the colorings $g_{1}$ and $g_{2}$ together constitute a cyclic interval 8-coloring of $G$.

We note that the above result is almost sharp, since there is an Eulerian bipartite graph with six vertices and maximum degree 12 without a cyclic interval coloring (see Fig. 3 in section 5). It is thus an interesting open problem if the condition of maximum degree at most 8 can be replaced by 10 in the above theorem.

Corollary 2.8 A bipartite graph $G$ where all vertex degrees are in the set $\{1,2,4,6,7,8\}$ has a cyclic interval coloring.

Proof. If $G$ is an Eulerian graph, the existence of a cyclic interval coloring is evident by Theorem 2.7. Suppose that $G$ has some vertices with odd degrees. Define an auxiliary graph $G^{\star}$ as follows: we take two isomorphic copies $G_{1}$ and $G_{2}$ of the graph $G$ and join by an edge every vertex with an odd vertex degree in $G_{1}$ with its copy in $G_{2}$. Clearly, $G^{\star}$ is a bipartite Eulerian graph with maximum degree at most 8 . Therefore, by Theorem 2.7. $G^{\star}$ has a cyclic interval coloring. It is not difficult to see that the restriction of this coloring to the edges of $G$ is a cyclic interval coloring of $G$.

Let us now consider $(a, b)$-biregular graphs. The following result is an evident corollary of Theorem 2.1 .

Corollary 2.9 If $G$ is a $(2 r-2,2 r)$-biregular $(r \geq 2)$ graph, then $G \in \mathfrak{N}_{c}$ and $w_{c}(G)=2 r$.

Our next result establishes a connection between the existence of cyclic interval colorings for $(a, b)$-biregular and $(a, b-1)$-biregular graphs.

Theorem 2.10 If every $(a, b)$-biregular $(a<b)$ graph has a cyclic interval $b$-coloring and $\operatorname{gcd}(a, b-1)=1$, then every $(a, b-1)$-biregular graph has a cyclic interval b-coloring.

Proof. Let $G$ be an $(a, b-1)$-biregular $(a<b)$ bipartite graph with bipartition $(X, Y)$. Clearly, $a|X|=(b-1)|Y|$. Since $\operatorname{gcd}(a, b-1)=1$, we have $|Y|=a k$ for some integer $k$. Let $Y=\left\{y_{1}, \ldots, y_{a k}\right\}$. Now we define an auxiliary graph $G^{\prime}$ as follows:

$$
V\left(G^{\prime}\right)=X \cup X^{\prime} \cup Y \text { and } E\left(G^{\prime}\right)=E(G) \cup E^{\prime},
$$

where $X^{\prime}=\left\{x_{1}^{\prime}, \ldots, x_{k}^{\prime}\right\}$ and $E^{\prime}=\left\{x_{i}^{\prime} y_{a(i-1)+1}, \ldots, x_{i}^{\prime} y_{a i}: 1 \leq i \leq k\right\}$. 
Clearly, $G^{\prime}$ is an $(a, b)$-biregular bipartite graph with bipartition $\left(X \cup X^{\prime}, Y\right)$, and since $G^{\prime}$ is $(a, b)$-biregular, $G^{\prime}$ has a cyclic interval $b$-coloring. It is not difficult to see that the restriction of this edge coloring to the edges of $G$ induces a cyclic interval $b$-coloring.

Since all $(3,6)$-biregular and $(4,8)$-biregular graphs have cyclic interval 6- and 8-colorings, respectively [8], we deduce the following two consequences from Theorem 2.10. The first one was first obtained in [7] (using essentially the same proof).

Corollary 2.11 [7] If $G$ is a $(3,5)$-biregular graph, then $G \in \mathfrak{N}_{c}$ and $w_{c}(G) \leq 6$.

Corollary 2.12 If $G$ is a $(4,7)$-biregular graph, then $G \in \mathfrak{N}_{c}$ and $w_{c}(G) \leq 8$.

\section{Cyclic interval colorings of outerplanar graphs}

Let us now consider outerplanar graphs. We conjecture that all connected outerplanar graphs have cyclic interval colorings, and we prove this conjecture for simple graphs with maximum degree at most 4 . For the proof, we shall use the fact that every simple 2connnected outerplanar graph with maximum degree 3 has an interval coloring with 3 or 4 colors [20].

Theorem 3.1 If $G$ is a simple connected outerplanar graph with maximum degree $\Delta(G) \leq$ 4 , then $G$ has a cyclic interval coloring.

Proof. If $\Delta(G) \leq 3$, then $G$ has a cyclic interval coloring by the result of Nadolski [19] so it suffices to prove the theorem when $\Delta(G)=4$.

We shall prove the theorem using the following two claims.

Claim 1 Every simple 2-connected outerplanar graph of maximum degree 4 has a cyclic interval 5-coloring.

Proof. Let $G$ be a simple 2-connected outerplanar graph. Then $G$ has a Hamiltonian cycle $C$, implying that $G-E(C)$ is a simple graph with maximum degree 2. We define a proper edge coloring $\alpha$ of $G-E(C)$ as follows: for each component which is a path or an even cycle we color the edges of it by colors 1 and 3 alternately; for each component which is an odd cycle we color one of the edges of it by color 2 , and the rest of the edges in the component by colors 1 and 3 alternately.

Suppose first that $|V(G)|$ is even; then we may properly color the edges of $C$ using colors 4 and 5. This edge coloring along with the coloring $\alpha$ of $G-E(C)$ constitute a cyclic interval coloring of $G$.

Suppose now that $|V(G)|$ is odd. We consider some different cases.

Case 1. $C$ contains two consecutive vertices both of which have degree 4 in $G$ :

Let $u$ and $v$ be two vertices of degree 4 in $G$, which are consecutive on $C$. Suppose first that $u$ and $v$ lie in different components of $G-E(C)$. Then we may without loss of 
generality assume that both $u$ and $v$ are incident with two edges colored 1 and 3 under $\alpha$, respectively (possibly by shifting colors along cycles or paths in $G-E(C)$ ). We define an edge coloring $\beta$ of $C$ by coloring $u v$ with color 2 and coloring the rest of the edges of $C$ by colors 4 and 5 alternately. It is straightforward that $\alpha$ and $\beta$ together constitute a cyclic interval coloring of $G$.

Suppose now that $u$ and $v$ belong to the same component $S$ of $G-E(C)$. Since $G$ is an outerplanar graph, this implies that $S$ is a path and thus its edges are colored by colors 1 and 3 under $\alpha$; we obtain a cyclic interval coloring by coloring the edges of $C$ as in the preceding case.

Case 2. $C$ does not contain two consecutive vertices both of which have degree 4 in $G$ :

Suppose first that there are vertices $u$ and $v$ of degree 3 and 4 in $G$, respectively, which are consecutive on $C$. If $u$ and $v$ lie in different components of $G-E(C)$, then we may assume that $v$ is incident with two edges colored 1 and 3, respectively, under $\alpha$. Moreover, $u$ is incident with an edge colored 1 or 3 under $\alpha$. We may thus proceed by coloring $u v$ with color 2 and then coloring the rest of $C$ by colors 4 and 5 alternately, starting with color 5 at $u$ if $u$ is incident with an edge colored 1 , and starting with color 4 at $u$ otherwise.

If $u$ and $v$ lie in the same component $S$ of $G-E(C)$, then $S$ is a path, and thus the edges of $S$ are colored alternately with colors 1 and 3 under $\alpha$. Thus, we may proceed as in the preceding paragraph.

Suppose now that there are no vertices $u$ and $v$ of degree 3 and 4 in $G$, respectively, which are consecutive on $C$. Then, if $u$ has degree 4, then any neighbor of $u$ on $C$ has degree 2. Suppose first that $G-E(C)$ contains at least one cycle or a path of length at least 3. Let $x$ be a vertex of degree 2 on this cycle or path, let $y$ be a neighbor of $x$ on $C$, and let $z$ be a neighbor of $x$ in $G-E(C)$ which has degree 2 in $G-E(C)$. We construct a proper edge coloring $\alpha^{\prime}$ of $G-E(C)$ from $\alpha$ by recoloring the edges of the component containing $x$ by colors $1,2,3$ in such a way that $x z$ is colored 2 , the other edge incident with $x$ is colored 1, and all other edges of this component is colored by 1 and 3 alternately. By coloring the edge $x y$ with color 3 , and the rest of the edges of $C$ by colors 4 and 5 , alternately, and starting with color 4 at $y$, we clearly obtain a cyclic interval coloring of $G$.

Suppose now that $G-E(C)$ is a vertex-disjoint union of paths all of which have length at most 2. Since $G$ is outerplanar and has maximum degree 4 in $G$, there is a path $P=x u v$ in $G-E(C)$ of length 2. Denote by $Q$ the path in $C$ with origin $u$ and terminus $v$ that contains $x$ as an inner vertex. We color $Q$ by colors 2 and 3 alternately, and starting with color 2 at $u$; we color all other edges of $C$ with colors 5 and 4 alternately and starting with color 5 at $u$. A component $S$ of $G-E(C)$ we color by 1 and 3 alternately if both endpoints of $S$ are in $V(C) \backslash V(Q)$; if the endpoints of $S$ are in $Q$ we color it by 1 and 4 alternately, except for the path $x u v$, where we color $x u$ with 1 and $u v$ with 3 (4) if $Q$ has odd (even) length. The resulting coloring is a cyclic interval 5-coloring.

We shall also need the following claim:

Claim 2 Every simple 2-connected outerplanar graph $G$ of maximum degree at most 3 has a cyclic interval 3-coloring $\alpha$ with the property that there is at most one vertex $v \in$ $V(G)$ such that $S_{G}(v, \alpha)$ is not an interval, or an interval coloring with at most 4 colors. 
Moreover, if $G$ is not interval colorable using at most 4 colors, then for any vertex $v \in$ $V(G)$ of degree 2 , we can choose the coloring $\alpha$ so that $v$ is the unique vertex with the property that $S_{G}(v, \alpha)$ is not interval.

Proof. If $G$ has maximum degree at most 2, then trivially $G$ has a cyclic interval coloring with the required property or an interval coloring (and thus also a cyclic interval coloring).

If $G$ has maximum degree 3, then the claim follows from the result of Petrosyan [20] that every simple 2-connected outerplanar graph of maximum degree 3 has an interval coloring with at most 4 colors.

We now finish the proof of the theorem by proving that every simple outerplanar graph with maximum degree 4 has a cyclic interval coloring. The proof is by induction on the number of blocks of $G$. Since any tree is interval colorable, we may assue that there is some cycle of $G$.

If $G$ has only one block, then the result follows from Claims 1 and 2. Suppose now that $G$ has two blocks $F_{1}$ and $F_{2}$, and that $v$ is the common vertex of these blocks. We shall prove that $G$ has a cyclic interval 5 -coloring.

Suppose first that one of the blocks, say $F_{2}$, consist of a single edge. Since any interval coloring with at most 5 colors is also a cyclic interval 5-coloring, it follows from Claims 1 and 2 that $F_{1}$ either has a

- cyclic interval 3-coloring $\alpha$, with the property that $v$ is the only vertex $u$ such that $S(u, \alpha)$ may not be an interval,

- a cyclic interval 5-coloring.

It is straightforward to verify that in both cases we may color the edge of $F_{2}$ to obtain a cyclic interval 5-coloring of $G$.

Suppose now that both $F_{1}$ and $F_{2}$ have maximum degree at least 2. Since $G$ has maximum degree 4 , this implies that two edges of $F_{1}$, and two edges of $F_{2}$, are incident with $v$. By Claims 1 and 2, for $i \in\{1,2\}, F_{i}$ either has a

- cyclic interval 3-coloring $\alpha_{i}$, with the property that $v$ is the only vertex $u$ such that $S\left(u, \alpha_{i}\right)$ may not be an interval, or

- a cyclic interval 5-coloring $\alpha_{i}$.

If both $F_{1}$ and $F_{2}$ have cyclic interval 3-colorings, $\alpha_{1}$ and $\alpha_{2}$, respectively, then we may assume that $S\left(v, \alpha_{1}\right)=S\left(v, \alpha_{2}\right)=\{1,3\}$, and we define a new coloring $\alpha_{2}^{\prime}$ of $F_{2}$ by setting $\alpha_{2}^{\prime}(e)=\alpha_{2}(e)+1$ for any edge $e$ of $F_{2}$. Taking $\alpha_{1}$ and $\alpha_{2}^{\prime}$ together we obtain an interval coloring of the graph, and hence a cyclic interval 5-coloring.

Suppose now that one of $F_{1}$ and $F_{2}$ has a cyclic interval 5-coloring. Assume e.g. that $\alpha_{1}$ is a cyclic interval 5 -coloring of $F_{1}$. Then we may rotate the colors of $\alpha_{1}$ modulo 5 to obtain a coloring $\alpha_{1}^{\prime}$ so that $S\left(v, \alpha_{1}^{\prime}\right) \cap S\left(v, \alpha_{2}\right)=\emptyset$, and thus $\alpha_{1}^{\prime}$ and $\alpha_{2}$ taken together form a cyclic interval 5-coloring.

Now assume that $G$ has several blocks $F_{1}, F_{2}, \ldots, F_{n}$ and that we have constructed a cyclic interval 5-coloring $\alpha$ of the connected subgraph $H_{r}$ of $G$ consisting of blocks 
$F_{1}, F_{2}, \ldots, F_{r}$. Suppose that $F_{r+1}$ has exactly one vertex in common with $H_{r}$. We complete the induction step by proving that there is a cyclic interval 5-coloring of the union $H_{r+1}$ of $H_{r}$ and $F_{r+1}$.

If $F_{r+1}$ consist of a single edge, then the result is trivial. Suppose now that $F_{r+1}$ has maximum degree at least 2 , and let $v$ be the common vertex of $H_{r}$ and $F_{r+1}$. It follows that 2 edges of $H_{r}$, and two edges of $F_{r+1}$, are incident with $v$. Moreover, by rotating the colors in $\alpha$ modulo 5, we may assume that $S_{H_{r}}(v, \alpha)=\{1,2\}$.

By Claims 1 and 2, $F_{r+1}$ has a cyclic interval 3-coloring $\beta$ satisfying that $v$ is the only vertex $u$ with the property that $S(u, \beta)$ may not be an interval, or a cyclic interval 5coloring. If the former holds, then by defining the coloring $\beta^{\prime}$ by setting $\beta^{\prime}(e)=\beta(e)+2$, we obtain a cyclic interval 5-coloring of $H_{r+1}$ by taking $\beta^{\prime}$ and $\alpha$ together. If $F_{r+1}$ has a cyclic interval 5 -coloring $\beta$, then by rotating the colors of $\beta$ we obtain a coloring $\beta^{\prime}$ such that $S\left(v, \beta^{\prime}\right) \cap S(v, \alpha)=\emptyset$, and thus $\alpha$ and $\beta^{\prime}$ together form a cyclic interval 5-coloring of $H_{r+1}$.

\section{Cyclic interval colorings of complete multipartite graphs}

A graph $G$ is called a complete $r$-partite $(r \geq 2)$ graph if its vertices can be partitioned into $r$ nonempty independent sets $V_{1}, \ldots, V_{r}$ such that each vertex in $V_{i}$ is adjacent to all the other vertices in $V_{j}$ for $1 \leq i<j \leq r$. Let $K_{n_{1}, n_{2}, \ldots, n_{r}}$ denote a complete $r$-partite graph with independent sets $V_{1}, V_{2}, \ldots, V_{r}$ of sizes $n_{1}, n_{2}, \ldots, n_{r}$. We set $n=\sum_{i=1}^{r} n_{i}$.

In [24], it was conjectured that all complete multipartite graphs are cyclically interval colorable. Here we prove this conjecture.

Theorem 4.1 For any $n_{1}, n_{2}, \ldots, n_{r} \in \mathbb{N}$, the graph $K_{n_{1}, n_{2}, \ldots, n_{r}}$ has a cyclic interval $n$ coloring.

Proof. We have that $n=\left|V\left(K_{n_{1}, n_{2}, \ldots, n_{r}}\right)\right|=\sum_{j=1}^{r} n_{j}$. For $0 \leq i \leq r$, define a sum $\sigma(i)$ as follows:

$$
\sigma(i)= \begin{cases}0, & \text { if } i=0, \\ \sum_{j=1}^{i} n_{j}, & \text { if } 1 \leq i \leq r .\end{cases}
$$

Clearly, $\sigma(r)=n$. Let $V_{i}=\left\{v_{\sigma(i-1)+1}, \ldots, v_{\sigma(i)}\right\}$ for $1 \leq i \leq r$.

Define an edge coloring $\alpha$ of $K_{n_{1}, n_{2}, \ldots, n_{r}}$ as follows: for $v_{i} v_{j} \in E\left(K_{n_{1}, n_{2}, \ldots, n_{r}}\right)$, let

$$
\alpha\left(v_{i} v_{j}\right)= \begin{cases}(i+j)(\bmod n), & \text { if } i+j \neq n \\ n, & \text { otherwise. }\end{cases}
$$

Let us prove that $\alpha$ is a cyclic interval $n$-coloring of $K_{n_{1}, n_{2}, \ldots, n_{r}}$.

First note that in the coloring $\alpha$ every color is used on some edge. Next let $v_{i} \in V_{l}$, where $1 \leq l \leq r$. If $l=1$, then, by the definition of $\alpha$, we have $S\left(v_{i}, \alpha\right)=[i+\sigma(1)+1, n] \cup[1, i]=$ $\left[i+n_{1}+1, n\right] \cup[1, i]$. If $1<l \leq r$, then, by the definition of $\alpha$, we have that $S\left(v_{i}, \alpha\right)$ contains colors $1, \ldots, n$ except for $i+\sigma(l-1)+1, \ldots, i+\sigma(l)$, where colors are taken modulo $n$ and with $n$ instead of 0 . This implies that $\alpha$ is a cyclic interval $n$-coloring of $K_{n_{1}, n_{2}, \ldots, n_{r}}$. 
Corollary 4.2 For any $n_{1}, n_{2}, \ldots, n_{r} \in \mathbb{N}$, we have $K_{n_{1}, n_{2}, \ldots, n_{r}} \in \mathfrak{N}_{c}$ and

$$
w_{c}\left(K_{n_{1}, n_{2}, \ldots, n_{r}}\right) \leq \sum_{i=1}^{r} n_{i} .
$$

We will show that the upper bound in Corollary 4.2 is sharp for some complete multipartite graphs. But first we need the following result.

Theorem 4.3 If for a graph $G$, there exists a number $d$ such that $d$ divides $d_{G}(v)$ for every $v \in V(G)$ and $d$ does not divide $|E(G)|$, then $G$ has no cyclic interval dk-coloring for every $k \in \mathbb{N}$.

Proof. Suppose, to the contrary, that $G$ has a cyclic interval $d k$-coloring $\alpha$ for some $k \in \mathbb{N}$. We call an edge $e \in E(G)$ a $d$-edge if $\alpha(e)=d l$ for some $l \in \mathbb{N}$. Since $d$ divides $d_{G}(v)$ for every $v \in V(G)$, and $\alpha$ is a cyclic interval coloring, we have that for any $v \in V(G)$, the set $S(v, \alpha)$ contains exactly $\frac{d_{G}(v)}{d} d$-edges. Now let $m_{d}$ be the number of $d$-edges in $G$. Then $m_{d}=\frac{1}{2} \sum_{v \in V(G)} \frac{d_{G}(v)}{d}=\frac{|E(G)|}{d}$. Hence, $d$ divides $|E(G)|$, which is a contradiction.

Corollary 4.4 If $G$ is an Eulerian graph and $|E(G)|$ is odd, then $G$ has no cyclic interval $t$-coloring for every even $t$.

Example 4.5 The graph consisting of three edge-disjoint triangles, where any two triangles have the same common vertex $v$, has a cyclic interval 7 -coloring: we color the first triangle by colors 1,2,3 so that 1 and 3 appear at $v$, color the second triangle by colors $2,3,4$ so that 2 and 4 appear at $v$, and we color the third triangle by colors $5,6,7$ so that 5 and 7 appear at $v$. This yields a cyclic interval 7 -coloring of the graph. However, by Corollary 4.4, this graph does not admit a cyclic interval 8-coloring.

The next result shows that the upper bound in Corollary 4.2 is sharp.

Corollary 4.6 If $r, n_{2}, \ldots, n_{r}$ and $\left|E\left(K_{1, n_{2}, \ldots, n_{r}}\right)\right|$ are odd, then

$$
w_{c}\left(K_{1, n_{2}, \ldots, n_{r}}\right)=1+\sum_{i=2}^{r} n_{i}
$$

Proof. Clearly, the graph $G=K_{1, n_{2}, \ldots, n_{r}}$ is Eulerian with the maximum degree $\Delta(G)=$ $\sum_{i=2}^{r} n_{i}$, which implies that $w_{c}(G) \geq \sum_{i=2}^{r} n_{i}$. By Corollary 4.4, $G$ has no cyclic interval $\Delta(G)$-coloring, since $|E(G)|$ is odd. This and Corollary 4.2 imply that $w_{c}(G)=1+$ $\sum_{i=2}^{r} n_{i}$. 


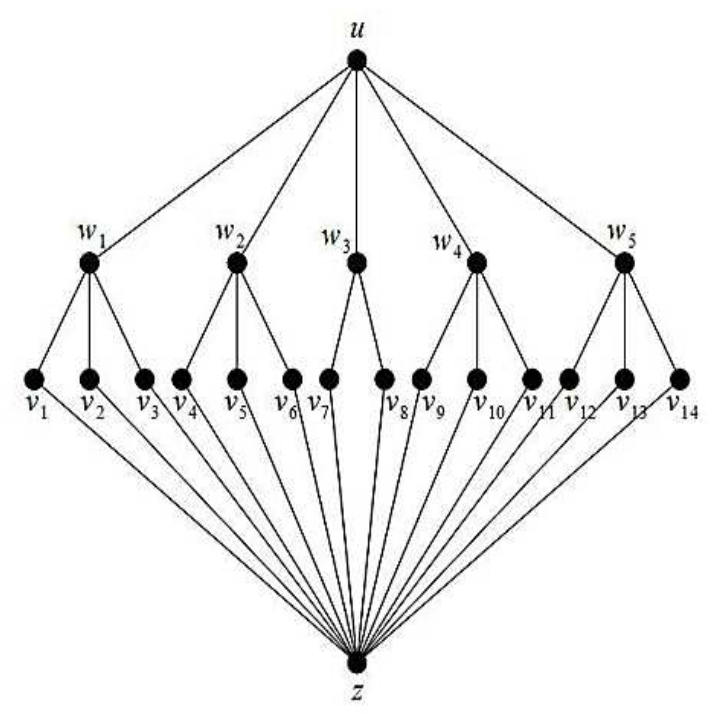

Figure 1. The cyclically interval non-colorable simple bipartite graph $G$.

\section{Bipartite graphs without cyclic interval colorings}

In this section we present some final observations; we give an example of a simple bipartite graph with maximum degree 14 without a cyclic interval coloring. In terms of maximum degree, this is an improvement of the smallest previously known bipartite example which has maximum degree 28 [19]. We also give a similar example for bipartite graphs with multiple edges. Our examples use graphs without interval colorings earlier constructed in [23]. For non-bipartite examples of graphs without cyclic interval colorings, see [24].

So let us consider the graph $G$ in Fig. 1. Clearly, $\Delta(G)=14$ and $|V(G)|=21$.

Proposition 5.1 The graph $G$ in Figure 1 is not cyclically interval colorable.

Proof. Suppose, to the contrary, that $G$ has a cyclic interval $t$-coloring $\alpha$ for some $t \geq 14$. Assume further that $\alpha$ is such a coloring using a minimum number of colors; then every color appears on at least one edge. We may assume that $S_{G}(u, \alpha)=[1,5]$ and $\alpha\left(u w_{j}\right)=i_{j}$ and $1 \leq i_{j} \leq 5$, for $j=1, \ldots, 5$. Let $N_{w_{j}}$ denote the neighbors of $w_{j}$ in $\left\{v_{1}, \ldots, v_{14}\right\}$ and consider the edges incident with $w_{j}$ and some vertex from $N_{w_{j}}$. Any such edge can receive colors only from the set $\left\{i_{j}-3, i_{j}-2, \ldots, i_{j}+3\right\}$, and therefore any edge joining $z$ with a vertex of $N_{w_{j}}$ can receive colors only from the set $\left\{i_{j}-4, i_{j}-3 \ldots, i_{j}+4\right\}$, where $(-)$ and $(+)$ denote subtraction modulo $t$ and addition modulo $t$, respectively. This implies that none of the edges incident with a vertex from $N_{w_{j}}$ is colored 10 .

Similarly, it can be shown that for any positive integer $\Delta \geq 14$, there exists a simple bipartite graph $G$ such that $G \notin \mathfrak{N}_{c}$ and $\Delta(G)=\Delta$. On the other hand, by Corollary 
2.4 every bipartite graph with maximum degree 4 has a cyclic interval 4 -coloring. So, it is natural to consider the following:

Problem 1 Is there a simple bipartite graph $G$ such that $5 \leq \Delta(G) \leq 13$ and $G \notin \mathfrak{N}_{c}$ ?

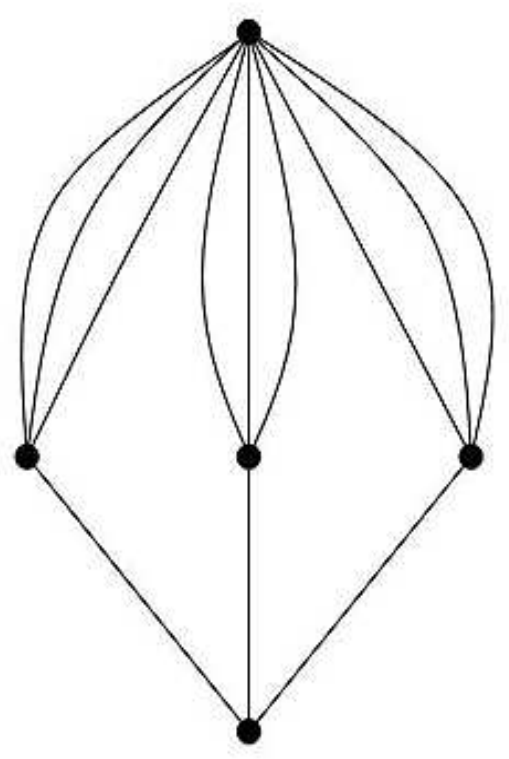

Figure 2. The cyclically interval non-colorable bipartite graph $H$.

Let us now consider the corresponding problem for bipartite graphs with multiple edges. It was proved in [23] that all bipartite graphs with at most four vertices have interval colorings; so all these graphs admit cyclic interval colorings. On the other hand, it is easy to see that the bipartite graph $H$ with $|V(H)|=5$ and $\Delta(H)=9$ shown in Fig. 2 has no cyclic interval coloring. We now prove a more general result.

Let us define graphs $H_{p, q}(p, q \in \mathbb{N})$ as follows: $V\left(H_{p, q}\right)=\left\{x, y_{1}, \ldots, y_{q}, z\right\}$ and $E\left(H_{p, q}\right)$ contains pairs of vertices $x$ and $y_{i}$, which are joined by $p$ edges and the edges $y_{i} z$ for $1 \leq i \leq q$. Clearly, $H_{p, q}$ is a connected bipartite graph with $\left|V\left(H_{p, q}\right)\right|=q+2, \Delta\left(H_{p, q}\right)=$ $d(x)=p q$, and $d(z)=q, d\left(y_{i}\right)=p+1, i=1, \ldots, q$. Fig. 3 shows the graph $H_{3,4}$.

Proposition 5.2 If $p q>2 p+q$, then $H_{p, q} \notin \mathfrak{N}_{c}$.

Proof. Suppose, to the contrary, that $H_{p, q}$ has a cyclic interval $t$-coloring $\alpha$ for some $t \geq$ $p q$. Assume further that $\alpha$ is such a coloring using a minimum number of colors; then every color appears on at least one edge. We may assume that $S(z, \alpha)=[1, q]$ and $\alpha\left(z y_{j}\right)=i_{j}$ 


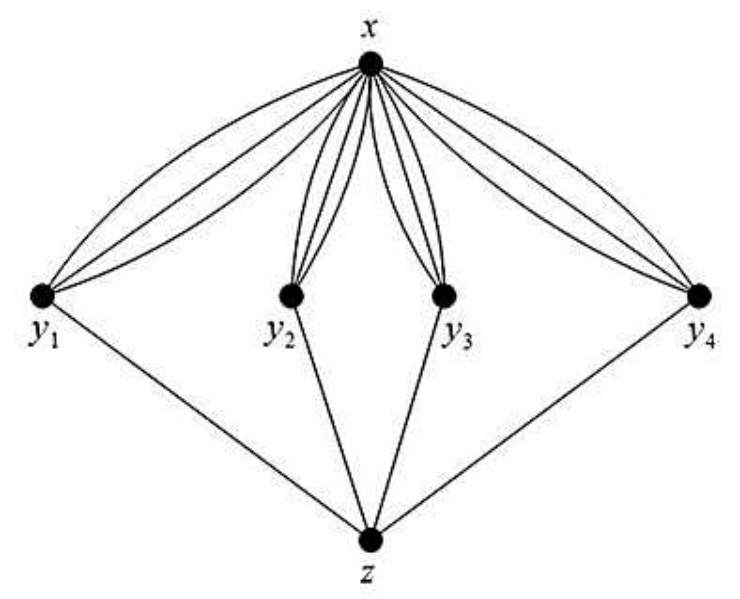

Figure 3. The graph $H_{3,4}$.

and $1 \leq i_{j} \leq q$, for $j=1, \ldots, q$. Consider the edges incident with the vertex $y_{j}$. Clearly, all parallel edges $x y_{j}$ can receive colors only from the set $\left\{i_{j}-p, i_{j}-p+1, \ldots, i_{j}+p\right\}$, where $(-)$ and $(+)$ denote subtraction modulo $t$ and addition modulo $t$, respectively. Since $p q>2 p+q$, we have that none of the edges $x y_{j}$ and $z y_{j}$ is colored $p q-p$.

Using similar arguments as in the proof of Proposition 5.2, it can be shown that for any positive integer $\Delta \geq 9$, there exists a bipartite graph $G$ such that $G \notin \mathfrak{N}_{c}$ and $\Delta(G)=\Delta$. So, it is natural to consider the following:

Problem 2 Is there a bipartite graph $G$ such that $5 \leq \Delta(G) \leq 8$ and $G \notin \mathfrak{N}_{c}$ ?

Acknowledgement The authors would like to thank the referees for their helpful comments and suggestions.

The third author would like to thank Linköping University for the hospitality and nice environment. The work of the third author was made possible by a research grant from the Armenian National Science and Education Fund (ANSEF) based in New York, USA.

\section{REFERENCES}

1. J. Akiyama and M. Kano, Factors and Factorizations of Graphs, proof techniques in Factor Theory, Springer-Verlag Berlin Heidelberg, 2011.

2. S. Altinakar, G. Caporossi and A. Hertz, On compact $k$-edge-colorings: A polynomial time reduction from linear to cyclic, Discrete Optimization 8 (2011), 502-512.

3. A.S. Asratian and C.J. Casselgren, On interval edge colorings of $(\alpha, \beta)$-biregular bipartite graphs, Discrete Math. 307 (2006), 1951-1956. 
4. A.S. Asratian, C.J. Casselgren, J. Vandenbussche and D.B. West, Proper path-factors and interval edge-coloring of (3, 4)-biregular bigraphs, J. Graph Theory 61 (2009), 8897.

5. A.S. Asratian and R.R. Kamalian, Interval colorings of edges of a multigraph, Appl. Math. 5 (1987) 25-34 (in Russian).

6. A.S. Asratian and R.R. Kamalian, Investigation on interval edge-colorings of graphs, J. Combin. Theory Ser. B 62 (1994), 34-43.

7. C.J. Casselgren, P.A. Petrosyan, B. Toft, On interval and cyclic interval edge colorings of $(3,5)$-biregular graphs, to appear in Discrete Mathematics.

8. C. J. Casselgren, B. Toft, On interval edge colorings of biregular bipartite graphs with small vertex degrees, J. Graph Theory 80 (2015), 83-97.

9. K. Giaro and M. Kubale, Consecutive edge-colorings of complete and incomplete Cartesian products of graphs, Congr. Numer. 128 (1997) 143-149.

10. K. Giaro and M. Kubale, Compact scheduling of zero-one time operations in multistage system, Discrete Applied Math. 145 (2004) 95-103.

11. H.M. Hansen, Scheduling with minimum waiting periods, MSc Thesis, Odense University, Odense, Denmark, 1992 (in Danish).

12. D. Hanson, C.O.M. Loten and B. Toft, On interval colorings of bi-regular bipartite graphs, Ars Combin. 50 (1998), 23-32.

13. R.R. Kamalian, Interval colorings of complete bipartite graphs and trees, preprint, Comp. Cen. of Acad. Sci. of Armenian SSR, Yerevan, 1989 (in Russian).

14. R.R. Kamalian, Interval edge colorings of graphs, Doctoral Thesis, Novosibirsk, 1990.

15. R.R. Kamalian and A.N. Mirumian, Interval edge-colorings of bipartite graphs of some class, Dokl. NAN RA 97 (1997), 3-5 (in Russian).

16. R.R. Kamalian, On cyclically-interval edge colorings of trees, Buletinul of Academy of Sciences of the Republic of Moldova, Matematica 1(68) (2012), 50-58.

17. R.R. Kamalian, On a number of colors in cyclically interval edge colorings of simple cycles, Open J. Discrete Math. 3 (2013), 43-48.

18. M. Kubale and A. Nadolski, Chromatic scheduling in a cyclic open shop, European J. Oper. Res. 164 (2005), 585-591.

19. A. Nadolski, Compact cyclic edge-colorings of graphs, Discrete Math. 308 (2008), 2407-2417.

20. P.A. Petrosyan, On Interval Edge-Colorings of Outerplanar Graphs, to appear in Ars Combinatoria

21. P.A. Petrosyan, Interval edge-colorings of complete graphs and $n$-dimensional cubes, Discrete Math. 310 (2010), 1580-1587.

22. P.A. Petrosyan, H.H. Khachatrian and H.G. Tananyan, Interval edge-colorings of Cartesian products of graphs I, Discuss. Math. Graph Theory 33(3) (2013), 613-632.

23. P.A. Petrosyan and H.H. Khachatrian, Interval non-edge-colorable bipartite graphs and multigraphs, J. Graph Theory 76 (2014), 200-216.

24. P.A. Petrosyan and S.T. Mkhitaryan, Interval cyclic edge-colorings of graphs, Discrete Mathematics 339 (2016), 1848-1860.

25. A.V. Pyatkin, Interval coloring of $(3,4)$-biregular bipartite graphs having large cubic subgraphs, J. Graph Theory 47 (2004), 122-128.

26. A.V. Pyatkin, On an interval $(1,1)$-coloring of incidentors of interval colorable graphs, 
Journal of Applied and Industrial Mathematics 9 (2015), 271-274.

27. S.V. Sevast'janov, Interval colorability of the edges of a bipartite graph, Metody Diskret. Analiza 50 (1990), 61-72 (in Russian).

28. D. de Werra and Ph. Solot, Compact cylindrical chromatic scheduling, SIAM J. Disc. Math, Vol. 4, N4 (1991), 528-534.

29. D. West, Introduction to Graph Theory, Prentice-Hall, New Jersey, 2001.

30. F. Yang and X. Li, Interval coloring of (3,4)-biregular bigraphs having two $(2,3)$ biregular bipartite subgraphs, Appl. Math. Let. 24 (2011), 1574-1577. 